\title{
Valor predictivo de la historia clínica y el examen físico en el diagnóstico de la neumonía del adulto adquirida en la comunidad. Revisión de la literatura
}

\author{
Fernando Saldías Pl, J Ignacio Méndez Ca, David Ramírez $\mathbf{R}^{\mathbf{a}}$, \\ O rlando Díaz $\mathbf{P}^{1}$. \\ Predictive value of history and \\ physical examination for the diagnosis \\ of community-acquired pneumonia \\ in adults. A literature review
}

Distinguishing pneumonia from other causes of respiratory illnesses, such as bronchitis, influenza and upper respiratory tract infections, has important therapeutic and prognostic implications. This decision is usually made by clinical assessment alone or by performing a chest x-ray. The reference standard for diagnosing pneumonia is chest radiography, but many physicians rely on history and physical examination to diagnose or exclude this disease. A review of published studies of patients suspected of having pneumonia reveals that there are no individual clinical findings, or combination of findings, that can predict with certainty the diagnosis of pneumonia. Prediction rules have been recommended to guide the order of diagnostic tests, to maximize their clinical utility. Thus, some studies have shown that the absence of any vital sign abnormalities or any abnormalities on chest auscultation substantially reduces the likelihood of pneumonia to a point where further diagnostic evaluation may be unnecessary. This article reviews the literature on the appropriate use of the history and physical examination in diagnose community-acquired pneumonia (Rev Méd Chile 2007; 135: 517-28).

(Key words: Diagnosis, differential; Pneumonia; Radiography, thoracic)

Recibido 19 de marzo, 2007. Aceptado el 22 de marzo, 2007.

Artículo preparado por invitación de los editores.

${ }_{1}^{1}$ Departamento de Enfermedades Respiratorias de la Pontificia Universidad Católica de Chile.

aAlumno de Medicina, Pontificia Universidad Católica de Chile. Santiago de Chile.

Correspondencia a: Dr. Fernando Saldías P. Departamento de Enfermedades Respiratorias, Pontificia Universidad Católica de Chile. Marcoleta 352, Santiago, Chile. Teléfonos: (562) 6331541-(562) 3543242. Fax: (562) 6335255. E mail: fsaldias@med.puc.cl 
$E^{n}$ Chile, las infecciones respiratorias agudas (resfríos, sinusitis, bronquitis, exacerbaciones de enfermedad pulmonar obstructiva crónica y asma bronquial, influenza y neumonía) constituyen el principal motivo de consulta en los servicios de atención primaria (Consultorios y Servicios de Urgencia), especialmente durante el período de otoño-invierno, coincidiendo con el aumento de las infecciones virales en la población (Figura $1)^{1-3}$. Las manifestaciones clínicas asociadas a las infecciones respiratorias, tales como malestar general, fiebre, calofríos, mialgias, odinofagia, rinorrea, tos, expectoración, dolor torácico y disnea, pueden presentarse en situaciones clínicas que difieren en su etiopatogenia, evolución, tratamiento y pronóstico ${ }^{4}$. Así, el cuadro clínico puede corresponder desde una infección respiratoria alta de origen viral autolimitada, hasta una infección del parénquima pulmonar que requiere tratamiento específico, como en los casos de neumonía y tuberculosis ${ }^{4,5}$.

Las infecciones respiratorias agudas son un problema prevalente, afectan a niños, adultos y ancianos; los principales patógenos involucrados son los virus respiratorios (rinovirus, influenza, parainfluenza, adenovirus, sincicial respiratorio) y, en segundo lugar, las bacterias (S pneumoniae, $\mathrm{H}$ influenzae, Mycoplasma pneumoniae, Chlamydia pneumoniae, etc.); son causa importante de ausentismo escolar y laboral, especialmente durante el periodo epidémico de otoño-invierno ${ }^{1-7}$.

El principal desafío diagnóstico que tienen los médicos de atención primaria es diferenciar a los pacientes con neumonía adquirida en la comunidad, que requieren tratamiento antibiótico, de otras afecciones respiratorias agudas, mayoritariamente de origen viral, que no requieren tratamiento específico, como resfríos, bronquitis e influenza ${ }^{1,2,4,5}$. Además, tienen la difícil tarea de balancear el problema diagnóstico determinado por los pacientes que consultan por fiebre o síntomas respiratorios agudos, evitando la prescripción indiscriminada de antibióticos en infecciones respiratorias virales, la que ha favorecido el desarmollo de microorganismos resistentes a los antimicrobianos ${ }^{8}$. En Chile, un tercio de las cepas de S pneumoniae muestra susceptibilidad disminuida a penicilina y en $18 \%$ de los casos la resistencia es alta (CIM $\geq 2 \mathrm{mg} / \mathrm{ml}$ ); mientras que la resistencia a enitromicina fluctúa entre 10\%-15\% y a cefotaxima entre $2 \%-10 \%$.
La neumonía adquirida en la comunidad es una enfermedad común, afecta principalmente a niños pequeños y ancianos, constituyendo una carga significativa para el sistema de salud de nuestro país ${ }^{2}$. En el enfrentamiento inicial del paciente que consulta por síntomas respiratorios agudos o fiebre, es necesario establecer el diagnóstico correcto basado en los elementos clínicos (anamnesis y examen físico) y de laboratorio (ej: hemograma, radiografía de tórax, proteína $\mathrm{C}$ reactiva, etc.) que se encuentran disponibles en la práctica ambulatoria. Para esto se requiere conocer la epidemiología de las infecciones respiratorias en el área geográfica donde está ubicado el Centro de Salud, junto con la sensibilidad y especificidad de los hallazgos de la historia clínica y examen físico para el diagnóstico de neumonía ${ }^{9}$. Una vez establecido el diagnóstico de neumonía, el médico debe evaluar la gravedad de la enfermedad mediante parámetros objetivos, lo cual le permite decidir el lugar más apropiado para su manejo (ambulatorio o en el hospital), la extensión de la evaluación diagnóstica y microbiológica, y las opciones de tratamiento antibiótico empírico ${ }^{10}$. Este artículo revisa el valor predictivo de la anamnesis, el examen físico y juicio clínico en el diagnóstico de la neumonía comunitaria del adulto; y realiza algunas recomendaciones que faciliten la evaluación diagnóstica y manejo inicial de los pacientes con infección respiratoria baja.

Entre los pacientes que consultan por fiebre 0 síntomas respiratorios agudos, es importante distinguir aquéllos que tienen una neumonía de quienes tienen otro diagnóstico (Tabla 1). Aunque la neumonía es poco frecuente en este contexto clínico (2\%-5\% de las consultas respiratorias), el riesgo de complicaciones y muerte asociados nos obliga a efectuar el diagnóstico y tratamiento apropiados ${ }^{5,11}$.

\section{EPIDEMIOLOGÍA DE LAS INFECCIONES RESPIRATORIAS}

Las infecciones respiratorias agudas constituyen el principal motivo de consulta ambulatoria en niños y adultos de ambos sexos, especialmente durante los meses de bajas temperaturas (Figura 1) ${ }^{1-3}$. Los niños pequeños (menores de 4 años) presentan 2 a 3 veces más infecciones respiratorias que la población adulta. Los principales microorganismos aislados son 


\section{Tabla 1. D iagnóstico diferencial de la neumonía comunitaria del adulto}

Causas frecuentes

Infecciones de la vía aérea superior

Bronquitis aguda

Influenza o gripe

Exacerbaciones de asma y EPOC

Tuberculosis pulmonar

Insuficiencia cardíaca congestiva

Tromboembolismo pulmonar

Enfermedad pulmonar neoplásica primaria y metastásica

Atelectasia pulmonar

Causas poco frecuentes

Neumonitis por hipersensibilidad

Daño pulmonar por drogas

Daño pulmonar por radioterapia

Linfangiosis carcinomatosa

Enfermedad colágeno-vascular: Lupus eritematoso, artritis reumatoidea, granulomatosis de

Wegener, síndrome de Churg-Strauss.

Sarcoidosis

Neumonía eosinofílica

Neumonía en organización criptogénica (COP)

los virus respiratorios, especialmente rinovirus (35\%), virus influenza A y B (30\%), virus parainfluenza (12\%), virus sincicial respiratorio (11\%) y adenovirus $(8 \%)^{12,13}$. Los principales mecanismos de contagio son la inhalación de aerosoles o microgotas con gérmenes y el contacto directo con secreciones infectadas (inoculación de las mucosas nasal, ocular o cavidad oral). El rinovirus, con sus diferentes serotipos (>100 serotipos), es el principal patógeno aislado en el resfrío común durante todo el año, con un aumento significativo de los casos en otoño y primavera ${ }^{14}$. La infección por el virus influenza es menos frecuente pero de mayor gravedad, determinando mayor número de consultas médicas y mayor ausentismo escolar y laboral ${ }^{3,7}$.

Las neumonías son afecciones inflamatorias agudas de origen infeccioso, ocasionadas por bacterias, virus $\mathrm{u}$ hongos, que comprometen el territorio alveolar ${ }^{1,3}$. La gravedad de la neumonía puede variar desde cuadros leves en personas sanas, que pueden confundirse con resfríos, bronquitis, gripe o infecciones de la vía aérea superior, hasta cuadros graves que deben ser manejados en unidades especializadas de cuidado intensivo y ponen en peligro la vida de los pacientes ${ }^{11}$.
Las enfermedades respiratorias constituyen la tercera causa de muerte de la población chilena, siendo sólo superadas por las enfermedades cardiovasculares y los tumores malignos ${ }^{2}$. Cincuenta por ciento de los decesos por causas respiratorias en el adulto es ocasionado por infecciones pulmonares o neumonías, elevándose sobre $80 \%$ en la población pediátrica. La incidencia, gravedad y letalidad de la neumonía se elevan en las edades extremas de la vida (menores de un año y mayores de 65 años) $)^{2,11}$. La incidencia de neumonía aumenta durante los meses de invierno, coincidiendo con el aumento de las infecciones respiratorias virales (Figura 1$)^{1-3}$.

Estudios de morbilidad estiman que la neumonía adquirida en la comunidad es el $4^{0}$ ó $5^{0}$ motivo de consulta ambulatoria de origen respiratorio en la población adulta ${ }^{3-5}$. La Tabla 1 describe los principales diagnósticos diferenciales de la neumonía comunitaria del adulto. Establecer el diagnóstico correcto de neumonía no es banal, considerando que es la única infección respiratoria aguda donde un retraso en el diagnóstico e inicio del tratamiento antibiótico se ha asociado a un mayor riesgo de complicaciones y muerte ${ }^{15-17}$. 


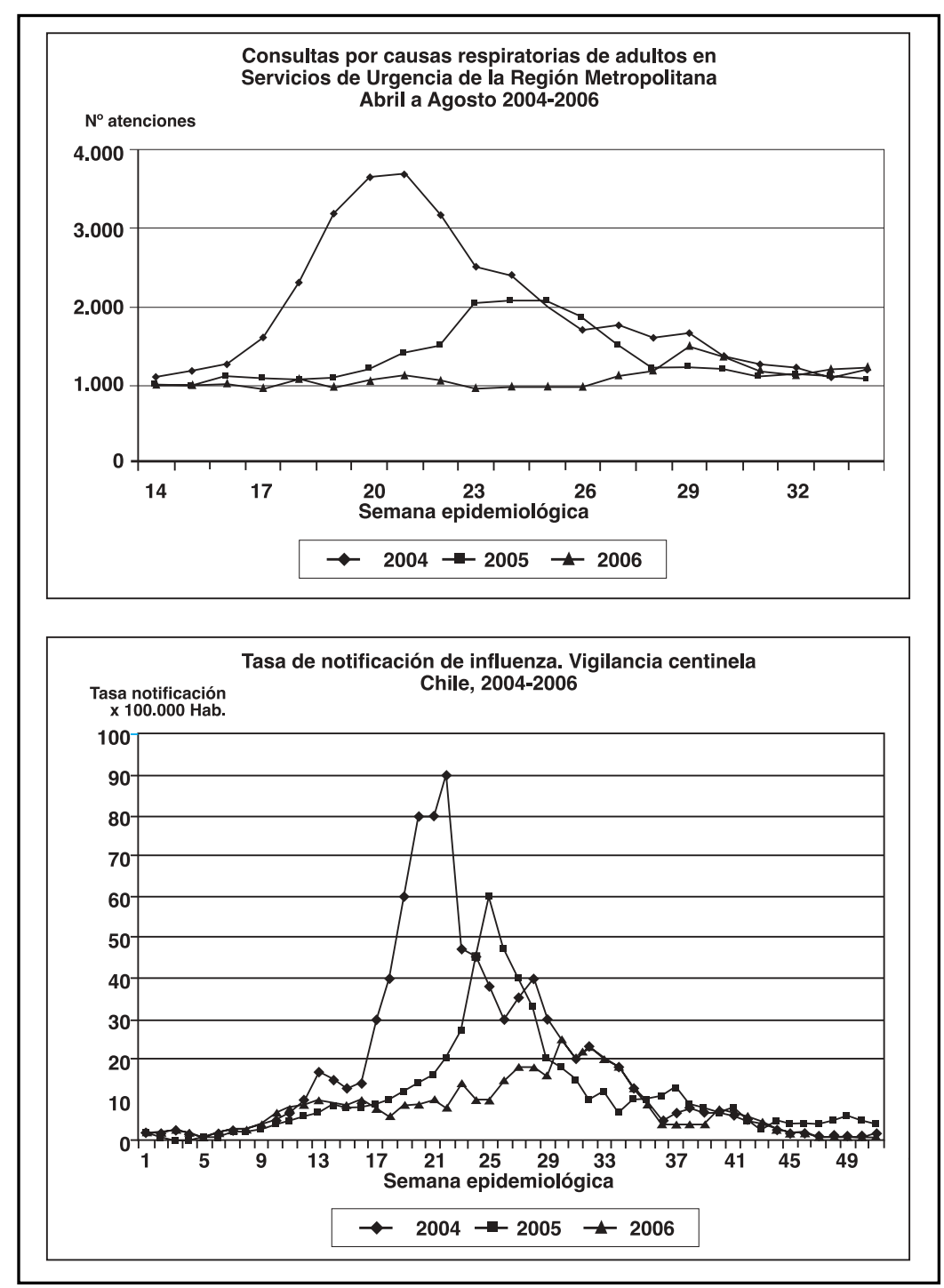

Figura 1. Consultas por causas respiratorias de adultos en Centros Centinela de la Región Metropolitana y tasa de notificación de influenza en Chile. Periodo 2004-2006².

\section{VALOR PREDICTIVO DE LA HISTORIA Y EXAMEN FÍSICO}

Los médicos de atención primaria confían en la historia clínica y el examen físico para confirmar o excluir el diagnóstico de neumonía. Sin embargo, los síntomas y signos tradicionalmente asociados con neumonía (ej: malestar general, fiebre, tos, expectoración, dolor torácico, disnea) no han demostrado ser buenos predictores en la práctica clínica habitual $^{9}$. La radiografía de tórax es a menudo solicitada por los clínicos para intentar diferenciar a los pacientes con neumonía de otras condiciones patológicas que comparten manifestaciones clínicas similares ${ }^{18}$

Algunos estudios sugieren que los médicos no realizan esta tarea de un modo eficiente. Bushyhead y cols examinaron la utilidad de la radiografía de tórax en la pesquisa de neumonía en dos mil pacientes con síntomas respiratorios, encontrando que sólo 32\% finalmente tenían neumonía 
y, basados sólo en el juicio clínico, casi la mitad de los pacientes con neumonía no habrían sido diagnosticados ni tratados en forma apropiada ${ }^{19}$. Las principales razones que se han mencionado para ello son: a) Los síntomas y signos clínicos son poco sensibles y específicos para establecer el diagnóstico, b) Existen deficiencias en la enseñanza de la semiología respiratoria, y c) La mayoría de los estudios han sido realizados en pacientes hospitalizados con neumonía comunitaria, en este grupo se concentraría el mayor riesgo de complicaciones y muerte, y sólo representan $20 \%-30 \%$ de los episodios de neumonía $9,11,20$. Además, los médicos en los Servicios de Urgencia suelen sobreestimar la probabilidad de neumonía de los enfermos que consultan por síntomas respiratorios $^{21}$.

La variabilidad interobservador en la pesquisa de los síntomas y signos clínicos en pacientes con neumonía no ha sido examinada en forma dirigida. Sin embargo, los estudios epidemiológicos de la prevalencia de síntomas respiratorios en la población han mostrado que existe considerable variabilidad interobservador en el registro de los síntomas, lo cual ha obligado a diseñar y utilizar cuestionarios estandarizados ${ }^{22,23}$. También se ha demostrado que existe gran variabilidad en la pesquisa de los signos físicos en pacientes con afecciones respiratorias agudas. Spiteri y cols, midieron la confiabilidad y precisión del examen pulmonar efectuado por 24 médicos, quienes examinaron a 24 pacientes con afecciones respiratorias variadas, incluyendo a cuatro pacientes con neumonía ${ }^{24}$. Como se describe en la Tabla 2, el grado de concordancia entre los médicos en la pesquisa de los hallazgos semiológicos fue variable y de magnitud moderada, con valores kappa que fluctuaron entre 0,01 y 0,52. Otros estudios han confirmado los mismos hallazgos. Schilling y $\operatorname{cols}^{25}$, encontraron $76 \%$ de concordancia en la pesquisa de ruidos respiratorios anormales en 187 pacientes con enfermedades pulmonares intersticiales y 88 sujetos control, con un valor de kappa de 0,25. Smyllie y cols, examinaron la habilidad clínica de 9 médicos que examinaron a 20 pacientes portadores de enfermedades respiratorias crónicas, encontrando grados moderados de concordancia en la pesquisa de matidez, disminución del murmullo pulmonar y crepitaciones ${ }^{26}$.

Sólo cinco estudios, incluyendo uno local, han examinado la sensibilidad, especificidad y valor predictivo de los hallazgos de la historia y examen físico en el diagnóstico de la neumonía del adulto adquirida en la comunidad (Tabla 3) ${ }^{27-31}$. Todos los estudios fueron prospectivos, realizados en una Unidad de Emergencia, los criterios de inclusión (fiebre o síntomas respiratorios) y el tamaño muestral fueron variables (examinaron entre $255 \mathrm{y}$ 1.819 adultos inmunocompetentes), el patrón de referencia para el diagnóstico de neumonía fue la presencia de un infiltrado pulmonar en la radiografía de tórax asociado o no a seguimiento clínico, y obtuvieron conclusiones similares.

Los criterios de selección de los pacientes fueron variables: Diehr y cols $^{27}$ examinaron una cohorte prospectiva de adultos que consultaron

Tabla 2. Evaluación de la precisión en la pesquisa de los hallazgos semiológicos en el examen de tórax²4

\begin{tabular}{|lcc|}
\hline Examen físico & Concordancia (\%) & Kappa \\
\hline Taquipnea & 63 & 0,25 \\
Cianosis & 68 & 0,36 \\
Disminución excursión torácica & 70 & 0,38 \\
Aumento frémito táctil & 85 & 0,01 \\
Matidez torácica & 77 & 0,52 \\
Disminución ruidos respiratorios & 71 & 0,43 \\
Sibilancias & 79 & 0,51 \\
Crepitaciones & 72 & 0,41 \\
Broncofonía & 81 & 0,32 \\
Pectoriloquia áfona & 71 & 0,11 \\
\hline
\end{tabular}


Tabla 3. Síntomas y signos clínicos asociados al diagnóstico de neumonía del adulto adquirida en la comunidad $\left(L R^{+}\right)^{27-31}$

\begin{tabular}{|c|c|c|c|c|c|}
\hline Variables & Diehr et al & Gennis et al & Singal et al & Heckerling & Saldías et al \\
\hline Tos & & NS & 1,8 & NS & NS \\
\hline Expectoración & 1,3 & NS & & NS & 1,2 \\
\hline Disnea & & 1,4 & NS & NS & NS \\
\hline Fiebre & 2,1 & NS & & 1,7 & 1,3 \\
\hline Escalofríos & 1,6 & 1,3 & -- & 1,7 & 1,4 \\
\hline Mialgias & 1,3 & NS & --- & & NS \\
\hline Odinofagia & 0,7 & NS & -1 & -- & 0,5 \\
\hline Rinorrea & 0,7 & NS & & & 0,5 \\
\hline $\mathrm{FC}>100 \mathrm{x}^{\prime}$ & NS & 1,6 & $\mathrm{NS}$ & 2,3 & 1,4 \\
\hline$F R>20 x^{\prime}$ & & 1,2 & & & 1,3 \\
\hline $\mathrm{T}^{\mathrm{o}}>37,8^{\circ} \mathrm{C}$ & 4,4 & 1,4 & 2,4 & 2,4 & 2,2 \\
\hline Signos vitales normales & & 0,2 & & & 0,3 \\
\hline Matidez & $\mathrm{NS}$ & 2,2 & ---- & 4,3 & 3,8 \\
\hline Roncus & NS & 1,5 & ----- & 1,4 & NS \\
\hline Broncofonía & & & & 3,5 & 9,5 \\
\hline Crepitaciones & 2,7 & 1,6 & 1,7 & 2,6 & 2,0 \\
\hline Examen pulmonar normal & - & 0,5 & -1 & - & 0,4 \\
\hline
\end{tabular}

LR+: razón de verosimilitud o likelihood ratio positivo. FC: frecuencia cardiaca, FR: frecuencia respiratoria, to: temperatura axilar. Signos vitales normales: frecuencia cardiaca $<100 \mathrm{lat} / \mathrm{min}$, frecuencia respiratoria $<20 \mathrm{resp} / \mathrm{min}$ y temperatura axilar $<37,8^{\circ} \mathrm{C}$. NS: variable no significativa.

en el Servicio de Urgencia por tos y le realizaron a todos una radiografía de tórax, encontrando una incidencia de neumonía de 2,6\%; mientras que los otros estudios evaluaron a una población seleccionada de pacientes que consultaron por síntomas respiratorios agudos o fiebre a quienes el médico de urgencia había decidido solicitar una radiografía de tórax, encontrando tasas más elevadas de neumonía (entre $12,4 \%$ y $38 \%$ ) $^{28-31}$. Por ende, la mayoría de los estudios publicados, con excepción de Diehr y cols, examinaron a una población seleccionada de pacientes con síntomas respiratorios agudos lo cual puede haber afectado la sensibilidad y especificidad de los hallazgos clínicos individuales. La prevalencia de neumonía en pacientes que consultan por síntomas respiratorios agudos es variable y depende de las características de la población y el lugar de atención, siendo más frecuente en los Servicios de Urgencia que en los consultorios de atención primaria ${ }^{1-5}$.

A pesar de sus diferencias metodológicas, los estudios demuestran que los elementos aislados de la historia y el examen físico no permiten establecer con seguridad el diagnóstico de neumonía (Tabla 3) 27-31. En general, los síntomas aislados fueron pobres predictores de neumonía $\left(\mathrm{LR}^{+}\right.$entre 0,5 y 2,1$)$, y no permiten confirmar ni excluir el diagnóstico sin solicitar una radiografía de tórax. Algo similar ocurrió con los signos clínicos, aunque la presencia de taquicardia, fiebre y algunas anomalías específicas en el examen pulmonar (matidez, broncofonia y crepitaciones) aumentaron moderadamente la probabilidad de neumonía. La razón de verosimilitud positiva $\left(\mathrm{LR}^{+}\right)$de cualquier anomalía en los signos vitales (taquicardia, taquipnea o fiebre) fluctuó entre $2 \mathrm{y}$ 4. Además, cuando se examinaron varios puntos de corte para estas anomalías no tuvieron impacto significativo en el cálculo de probabilidades. En dos estudios se ha comunicado que la presencia de signos vitales y auscultación pulmonar normales disminuyen en forma significativa la probabilidad de neumonía 28,31 . De este modo, si la prevalencia de neumonía en pacientes ambulato- 
rios que consultan por síntomas respiratorios es cercana a $5 \%$, los pacientes con signos vitales normales tendrían una probabilidad de neumonía inferior a $1 \%-2 \%$.

Los estudios clínicos concluyeron que la presencia o ausencia de los síntomas y signos clásicos asociados al diagnóstico de neumonía no permiten confirmar ni excluir con seguridad el diagnóstico sin solicitar un examen radiográfico del tórax ${ }^{27-31}$. En el estudio nacional, los principales predictores clínicos de neumonía fueron la fiebre $\left(\geq 38^{\circ} \mathrm{C}\right)$, taquipnea $(\geq 20 \mathrm{resp} / \mathrm{min})$, confusión mental, ortopnea, cianosis, tiraje, matidez torácica, broncofonia, crepitaciones y $\mathrm{SaO}_{2}$ menor de $90 \%$ respirando aire ambiente $\left(\mathrm{LR}^{+}: 2,0-9,5\right)^{31}$. La odinofagia, rinorrea, signos vitales y auscultación pulmonar normales fueron menos frecuentes en los pacientes con diagnóstico final de neumonía ( $\mathrm{LR}^{+}:$0,3-0,5). A diferencia de otros estudios, Saldías y cols. examinaron algunas combinaciones de síntomas y signos demostrando que se incrementa significativamente la probabilidad pretest. La Tabla 4 describe algunas combinaciones de variables clínicas que incrementan la probabilidad de neumonía, tales como fiebre y taquipnea asociados a ortopnea, matidez torácica, crepitaciones $0 \mathrm{SaO}_{2}$ menor de 90\% (LR+: 4,9-14,7). La probabilidad de neumonía es muy baja en los pacientes que consultan por síntomas respiratorios y tienen signos vitales y examen pulmonar normales (LR: 0,1$)$.

\section{EL JUICIO CLíNICO Y LAS GUÍAS DE DECISIÓN}

Aunque los médicos suelen planificar el estudio diagnóstico y la solicitud de exámenes complementarios considerando la prevalencia de la enfermedad y su estimación de la probabilidad en la población que están evaluando, el umbral diag-

Tabla 4. Valor predictivo de algunas combinaciones de variables clínicas asociadas al diagnóstico de la neumonía del adulto adquirida en la comunidad ${ }^{31}$

\begin{tabular}{|c|c|c|c|c|c|c|}
\hline Variables clínicas & Sensibilidad & Especificidad & VPP & VPN & $\mathrm{LR}^{+}$ & $\mathrm{p}$ \\
\hline Fiebre + escalofríos & $51 \%$ & $66 \%$ & $44 \%$ & $72 \%$ & 1,5 & 0,003 \\
\hline \multicolumn{7}{|l|}{ Fiebre + escalofríos } \\
\hline + expectoración & $40 \%$ & $81 \%$ & $52 \%$ & $72 \%$ & 2,1 & 0,001 \\
\hline Fiebre $+F R \geq 30 x^{\prime}$ & $24 \%$ & $92 \%$ & $63 \%$ & $69 \%$ & 3,2 & 0,001 \\
\hline \multicolumn{7}{|l|}{ Fiebre $+F R \geq 30 x^{\prime}$} \\
\hline + ortopnea & $7 \%$ & $99 \%$ & $89 \%$ & $66 \%$ & 14,7 & 0,001 \\
\hline $\mathrm{FR} \geq 20 \mathrm{x}^{\prime}+\mathrm{T} \geq 38^{\circ} \mathrm{C}$ & $32 \%$ & $88 \%$ & $58 \%$ & $71 \%$ & 2,6 & 0,001 \\
\hline \multicolumn{7}{|l|}{$\mathrm{FR} \geq 20 \mathrm{x}^{\prime}+\mathrm{T} \geq 38^{\circ} \mathrm{C}$} \\
\hline + matidez & $4 \%$ & $99 \%$ & $80 \%$ & $66 \%$ & 7,5 & 0,04 \\
\hline \multicolumn{7}{|l|}{$\mathrm{FR} \geq 20 \mathrm{x}^{\prime}+\mathrm{T} \geq 38^{\circ} \mathrm{C}$} \\
\hline + crepitaciones & $20 \%$ & $96 \%$ & $72 \%$ & $69 \%$ & 4,9 & 0,001 \\
\hline \multicolumn{7}{|l|}{$\mathrm{FR} \geq 20 \mathrm{x}^{\prime}+\mathrm{T} \geq 38{ }^{\circ} \mathrm{C}$} \\
\hline$+\mathrm{SaO}_{2}<90 \%$ & $8 \%$ & $99 \%$ & $89 \%$ & $66 \%$ & 14,6 & 0,001 \\
\hline Signos vitales normales & $14 \%$ & $56 \%$ & $14 \%$ & $55 \%$ & 0,3 & 0,001 \\
\hline \multicolumn{7}{|l|}{ Signos vitales + examen } \\
\hline pulmonar normales & $2 \%$ & $81 \%$ & $5 \%$ & $61 \%$ & 0,1 & 0,001 \\
\hline Odinofagia + Rinorrea & $9 \%$ & $74 \%$ & $15 \%$ & $61 \%$ & 0,3 & 0,001 \\
\hline \multicolumn{7}{|l|}{ Odinofagia + Rinorrea } \\
\hline + examen pulmonar normal & $1 \%$ & $88 \%$ & $4 \%$ & $63 \%$ & 0,1 & 0,001 \\
\hline
\end{tabular}

VPP: Valor predictivo positivo, VPN: Valor predictivo negativo, LR: Likelihood ratio, FR: Frecuencia respiratoria, to: Temperatura axilar, $\mathrm{SaO}_{2}$ : Saturación arterial de oxígeno. Signos vitales normales: frecuencia cardiaca $<100 \mathrm{lat} / \mathrm{min}$, frecuencia respiratoria $<20 \mathrm{resp} /$ min y temperatura axilar $<37,8^{\circ} \mathrm{C}$. 
nóstico de los profesionales varía considerablemente incluso cuando se ven enfrentados a situaciones clínicas semejantes ${ }^{32,33}$. Como el valor predictivo de los síntomas y signos clínicos aislados para el diagnóstico de neumonía es relativamente bajo, para resolver este problema se diseñaron algunas reglas predictivas o guías de decisión que incorporan la presencia o ausencia de hallazgos semiológicos específicos y tienen como propósito orientar a los clínicos en el manejo de los enfermos con características clínicas similares ${ }^{9}$. En la literatura médica encontramos varios protocolos o reglas de decisión que han sido diseñados, específicamente, para ayudar a los médicos de atención primaria en la pesquisa de pacientes adultos con neumonía adquirida en la comunidad (Tabla 5) ${ }^{27-30}$. Así, en las guías de práctica clínica, se suele recomendar a los médicos de atención primaria solicitar radiografía de tórax en los pacientes que consultan por síntomas respiratorios, basados en algunas de estas reglas de decisión, con el propósito de optimizar el cuidado de los enfermos ${ }^{34-36}$.

En las reglas predictivas descritas por Diehr y Heckerling, se modifica la probabilidad pretest de neumonía según estén presentes o ausentes ciertos hallazgos semiológicos específicos ${ }^{27,30}$. Mientras que la regla de Singal y cols fue diseñada mediante un análisis de regresión logística y entrega una probabilidad de neumonía que fluctúa entre 4\% (ausencia de síntomas y signos) y $49 \%$ (presencia de tos, fiebre y crepitaciones) $^{29}$. Gennis y cols, sugieren solicitar radiografía de tórax a los pacientes con alteración en cualquiera de los signos vitales (frecuencia cardiaca mayor de 100 latidos/min, frecuencia respiratoria mayor de $20 \mathrm{resp} / \mathrm{min}$ o temperatura mayor de $\left.37,8^{\circ} \mathrm{C}\right)^{28}$.

En dos estudios prospectivos se examinó el valor predictivo del juicio clínico y las cuatro reglas predictivas en el diagnóstico de neumonía

\section{Tabla 5. D escripción de las guías de decisión clínica para la solicitud de radiografía de tórax y pesquisa de neumonía adquirida en la comunidad en adultos con síntomas respiratorios que consultan en los servicios de urgencia}

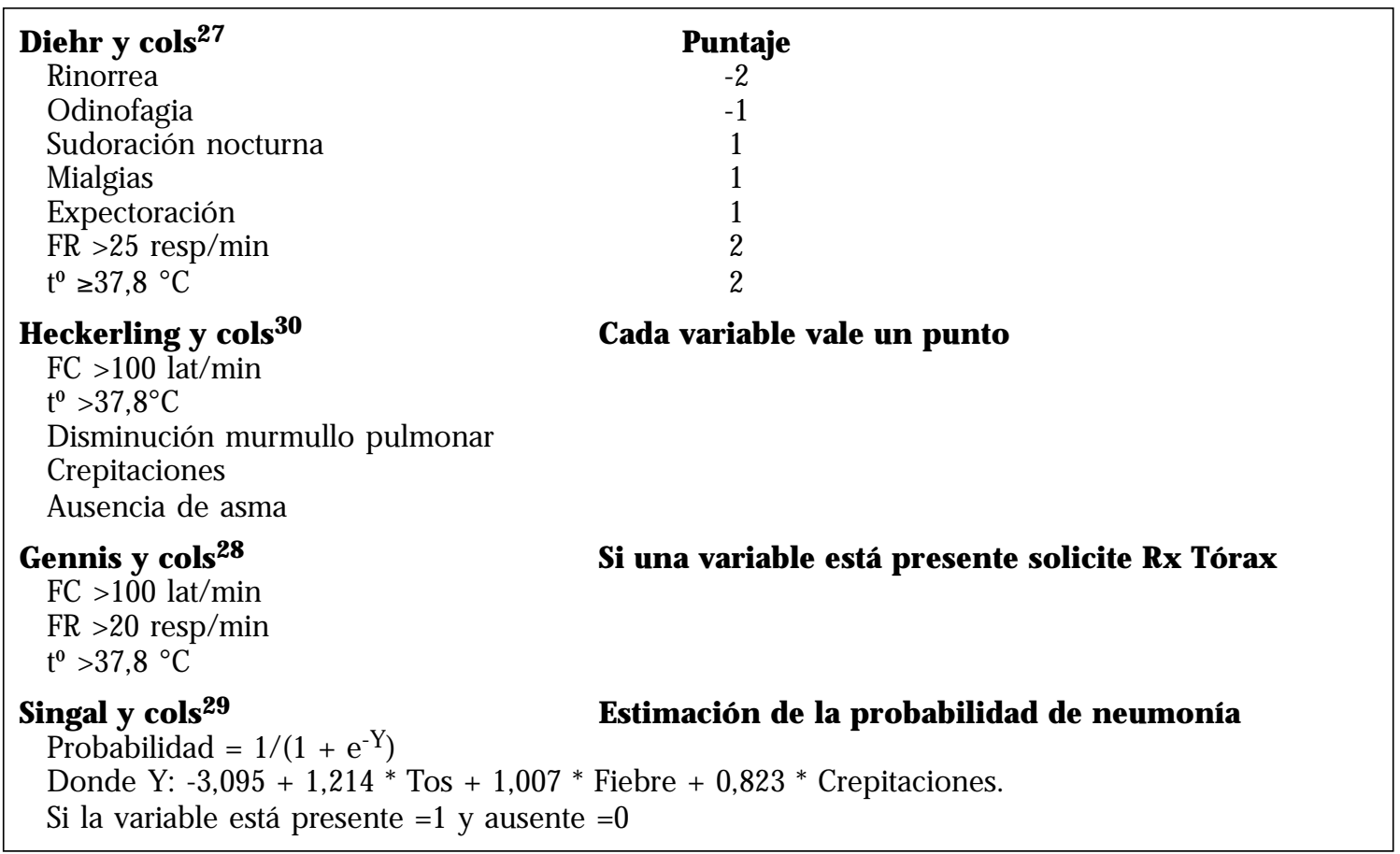

FC: frecuencia cardiaca, FR: frecuencia respiratoria, to: temperatura. Rx Tórax: radiografía de tórax. 
comunitaria $^{37,38}$. Emerman y cols, evaluaron el diagnóstico clínico de neumonía realizado por los médicos de la unidad de emengencia y una unidad de atención ambulatoria, en pacientes que consultaron por tos ${ }^{37}$. La prevalencia de neumonía en este estudio fue $7 \%$ y encontraron que el juicio clínico permitía reducir la solicitud de radiografías de tórax innecesarias mejor que las cuatro reglas predictivas ( $\mathrm{LR}^{-}=0,25$, IC 95\%: 0,09-0,61), mientras que el juicio clínico permitió aumentar la probabilidad de neumonía a sólo $13 \%\left(\mathrm{LR}^{+}=2,0\right.$, IC $95 \%$ : 1,5-2,4), lo cual habría conducido a solicitar muchos exámenes radiográficos innecesarios.

Entre las cuatro reglas predictivas examinadas, la solicitud de radiografía de tórax sólo a los pacientes con anomalías en los signos vitales, recomendada por Gennis y cols, tuvo mayor rendimiento diagnóstico con $\mathrm{LR}^{+}$de 2,6 (IC 95\%: 1,6-3,7), lo cual habría reducido en $40 \%$ la solicitud de exámenes radiográficos innecesarios pero no se habrían detectado $38 \%$ de las neumonías confirmadas por radiología ( $\mathrm{LR}^{-}=0,50$, IC 95\%: 0,27-0,78 comparado con $\mathrm{LR}^{-}=0,18$ del estudio original de Gennis y cols) ${ }^{37}$. La sensibilidad del juicio clínico (86\%) fue superior a las cuatro reglas de decisión (62\%-76\%), pero las reglas descritas por Diehr (67\%), Heckerling (67\%) y Gennis (76\%) fueron más específicas que el juicio clínico (58\%).

En el estudio de Saldías y cols, el diagnóstico clínico de neumonía realizado por los médicos en la unidad de emergencia tuvo mejor sensibilidad (rango: 75\%-83\%) que especificidad (rango: 47\%83\%), y mejor valor predictivo negativo (rango: 85\%-91\%) que positivo (rango: 36\%-70\%) ${ }^{31,38}$. En el diagnóstico de neumonía, el médico de urgencia menos experimentado tuvo menor valor predictivo positivo y especificidad comparado con los médicos especialistas en medicina interna y enfermedades respiratorias (Tabla 6). La probabilidad de modificar el diagnóstico inicial de neumonía o la razón de verosimilitud $\left(\mathrm{LR}^{+}\right)$del juicio clínico de los tres médicos de urgencia varió entre 1,5 y 4,8. Hallazgos similares fueron descritos por Wipf y cols, quienes evaluaron la precisión y variabilidad de los examinadores en la pesquisa de los signos clínicos específicos en pacientes con y sin diagnóstico de neumonía ${ }^{39}$. Los autores concluyeron que los hallazgos semiológicos pesquisados en el examen de tórax no permiten confirmar ni excluir con seguridad el diagnóstico de neumonía, existiendo gran variabilidad interobservador en la pesquisa de hallazgos semiológicos específicos (Tabla 7). González y cols, en un estudio prospectivo realizado en dos Servicios de Urgencia en Madrid, señalaron que el juicio clínico tiene baja sensibilidad para el diagnóstico de neumonía (45\%) con un moderado valor predictivo positivo $(80 \%)^{40}$. La sensibilidad y especificidad del diagnóstico clínico de neumonía establecido por los médicos de la Unidad de Emergencia fueron similares o ligeramente superior comparados con los cuatro índices clínicos descritos en la literatura ${ }^{37,38}$. El área bajo la curva (AUC) del juicio clínico y la guía de decisión descrita por Diehr y cols, fueron similares (AUC $=0,79$ y 0,75 , respectivamente), y ambos fueron superiores a los predictores clínicos descritos por Heckerling (AUC $=0,70$ ), Singal (AUC $=0,70$ ) y Gennis (AUC $=0,67)^{38}$.

En resumen, los hallazgos de la historia y el examen físico tienen moderada sensibilidad $\mathrm{y}$ especificidad para establecer el diagnóstico de neumonía en adultos inmunocompetentes que

Tabla 6. Sensibilidad, especificidad, valor predictivo positivo (VPP) y negativo (VPN) y razón de verosimilitud $\left(\mathrm{LR}^{+}\right)$del juicio clínico para la pesquisa de neumonía adquirida en la comunidad ${ }^{31}$

\begin{tabular}{|lccccc|}
\hline Juicio clínico & Sensibilidad & Especificidad & VPP & VPN & $\mathrm{LR}^{+}$ \\
\hline Médico A & $83 \%$ & $83 \%$ & $70 \%$ & $91 \%$ & 4,8 \\
Médico B & $75 \%$ & $73 \%$ & $56 \%$ & $86 \%$ & 2,8 \\
Médico C & $77 \%$ & $47 \%$ & $36 \%$ & $85 \%$ & 1,5 \\
Promedio & $79 \%$ & $66 \%$ & $55 \%$ & $85 \%$ & 2,3 \\
\hline
\end{tabular}

Médicos A y B: especialistas en medicina interna y enfermedades respiratorias con más de 5 años de ejercicio profesional, Médico C: especialista en medicina de urgencia con menos de 3 años de ejencicio profesional. 


\section{Tabla 7. Valor predictivo del juicio clínico en el diagnóstico de la neumonía del adulto adquirida en la comunidad ${ }^{37-40}$}

\begin{tabular}{|lrrrrr|}
\hline Juicio clínico & Sensibilidad & Especificidad & VPP & VPN & LR $^{+}$ \\
\hline González et al. & $45 \%$ & $93 \%$ & $80 \%$ & $74 \%$ & 6,6 \\
Wipf et al. & $47-69 \%$ & $58-75 \%$ & $48-64 \%$ & $57-72 \%$ & $1,1-2,0$ \\
Emerman et al. & $86 \%$ & $58 \%$ & $14 \%$ & $98 \%$ & 2,0 \\
Lieberman et al. & $49-90 \%$ & $78-88 \%$ & $16-42 \%$ & $94-99 \%$ & --- \\
Saldías et al. & $75-83 \%$ & $47-83 \%$ & $36-70 \%$ & $85-91 \%$ & $1,5-4,8$ \\
\hline
\end{tabular}

VPP: Valor predictivo positivo, VPN: Valor predictivo negativo, LR: Likelihood ratio.

consultan por fiebre o síntomas respiratorios agu$\operatorname{dos}^{27-31,37-41}$. Ninguna de las reglas de decisión descritas en la literatura han sido superiores al juicio clínico en el diagnóstico de neumonía, y aún no existen estudios que hayan examinado su real aporte en la evaluación y manejo de los pacientes que consultan por síntomas respiratorios o fiebre en los servicios de asistencia ambulatoria.

\section{ReCOMENDACiONES DEL CONSENSO NACIONAL DE NeUmonía Comunitaria ${ }^{36}$}

El diagnóstico clínico de neumonía sin confirmación radiográfica carece de precisión ya que el cuadro clínico (historia y examen físico) no

\section{REFERENCIAS}

1. Garibaldi RA. Epidemiology of community-acquired respiratory tract infections in adults: incidence, etiology, and impact. Am J Med 1985; 78: 32-7.

2. Ministerio de Salud de Chile. Programa de Infecciones Respiratorias Agudas (IRA), Departamento de Estadísticas e Información de Salud, Ministerio de Salud de Chile (http: // deis.minsal. cl/ index.asp)

3. FILE TM. The epidemiology of respiratory tract infections. Semin Respir Infect 2000; 15: 184-94.

4. González R, Steiner JF, SAnde MA. Antibiotic prescribing for adults with colds, upper respiratory tract infections, and bronchitis by ambulatory care physicians. JAMA 1997; 278: 901-4. permite diferenciar con certeza al paciente con neumonía de otras condiciones respiratorias agudas (infecciones de la vía aérea superior, bronquitis, influenza). El diagnóstico de neumonía basado exclusivamente en criterios clínicos también se ve dificultado por la gran variabilidad en la capacidad de detectar signos focales en el examen de tórax entre distintos observadores $^{24,26,39}$.

El diagnóstico de la neumonía comunitaria del adulto es clínico-radiográfico: la historia y examen físico sugieren la presencia de una infección del tracto respiratorio inferior, pero el diagnóstico de certeza se establece cuando se demuestra la presencia de infiltrados pulmonares de aparición reciente en la radiografía de tórax ${ }^{18,19}$.

5. Metlay JP, Stafford RS, Singer DE. National trends in the use of antibiotics by primary care physicians for adult patients with cough. Arch Intern Med 1998; 158: 1813-18.

6. Jackson ML, Neuzil KM, Thompson WW, Shay DK, Yu O, HANSON CA ET AL. The burden of community-acquired pneumonia in seniors: results of a population-based study. Clin Infect Dis 2004; 39: 1642-50.

7. CRighton EJ, Moineddin R, Mamdani M, Upshur RE. Influenza and pneumonia hospitalizations in Ontario: a time-series analysis. Epidemiol Infect 2004; 132: 1167-74.

8. Saldías F, Flores LJ, Torres C, García P, Díaz A. Susceptibilidad a antimicrobianos de Streptococcus pneumoniae en población infantil y adulta de 
Santiago. Período 1997-2003. Rev Méd Chile 2005; 133: 42-9.

9. Metlay JP, Kapoor WN, Fine MJ. Does this patient have community-acquired pneumonia? Diagnosing pneumonia by history and physical examination. JAMA 1997; 278: 1440-5.

10. Saldías F, Mardonez JM, Marchesse M, Viviani $P$, FARíAs G, Díaz A. Neumonía adquirida en la comunidad en el adulto hospitalizado. Cuadro clínico y factores pronósticos. Rev Méd Chile 2002; 130: 1373-82.

11. Fine MJ, Smith MA, Carson CA, Mutha SS, Sankey SS, WeisfFeld LA et al. Prognosis and outcomes of patients with community-acquired pneumonia. A meta-analysis. JAMA 1996; 275: 134-41.

12. GREENBERG SB. Respiratory viral infections in adults. Curr Opin Pulm Med 2002; 8: 201-8.

13. Monto AS. Epidemiology of viral respiratory infections. Am J Med 2002; 112 (Suppl 6A): 4S-12S.

14. FendRICK AM. Viral respiratory infections due to rhinoviruses: current knowledge, new developments. Am J Ther 2003; 10: 193-202.

15. Meehan TP, Fine MJ, Krumholz HM, Scinto JD, Galusha DH, MockaLS JT ET aL. Quality of care, process, and outcomes in elderly patients with pneumonia. JAMA 1997; 278: 2080-4.

16. BatTleman DS, CaLdahan M, Thaler HT. Rapid antibiotic delivery and appropriate antibiotic selection reduce length of hospital stay of patients with community-acquired pneumonia: link between quality of care and resource utilization. Arch Intern Med 2002; 162: 682-8.

17. Houck PM, Bratzler DW, Nsa W, Ma A, Bartlett JG. Timing of antibiotic administration and outcomes for Medicare patients hospitalized with community-acquired pneumonia. Arch Intern Med 2004; 164: 637-44.

18. Katz DS, Leung AN. Radiology of pneumonia. Clin Chest Med 1999; 20: 549-62.

19. Bushy head JB, Wood RW, Tompkins RK, Wolcott BW, DieHR P. The effect of chest radiographs on the management and clinical course of patients with acute cough. Med Care 1983; 21: 661-73.

20. ZEIHER B, Dawson NV. Spectrum and bias in the assessment of adult outpatient pneumonitis. Clin Res 1986; 34: 1016A.

21. Christensen-Szalanski JJ, Bushyhead JB. Physician's use of probabilistic information in a real clinical setting. J Exp Psychol Hum Percept Perform 1981; 7: 928-35.
22. Cochrane AL, Chapman PJ, Oldham PD. Observers' errors in taking medical histories. Lancet 1951; 1: 1007-9.

23. FLETCHER CM. The problem of observer variation in medical diagnosis with special reference to chest diseases. Methods Inf Med 1964; 3: 98-103.

24. SPITERI MA, Cook DG, Clarke SW. Reliability of eliciting physical signs in examination of the chest. Lancet 1988; 1: 873-5.

25. Schimng RS, Hughes JP, Dingwal-Fordyce I. Disagreement between observers in an epidemiological study of respiratory disease. BMJ 1955; 1: 65-8.

26. Smywe HC, Biendis LM, Armtage P. Observer disagreement in physical signs of the respiratory system. Lancet 1965; 40: 412-3.

27. Diehr P, Wood RW, Bushyhead J, Krueger L, Wolcott B, Tompkins RK. Prediction of pneumonia in outpatients with acute cough - a statistical approach. J Chron Dis 1984; 37: 215-25.

28. Gennis P, GaLiagher J, Falvo C, Baker S, Than W. Clinical criteria for the detection of pneumonia in adults: guidelines for ordering chest roentgenograms in the emergency department. J Emerg Med 1989; 7: 263-8.

29. Singal BM, Hedges JR, Radack KL. Decision rules and clinical prediction of pneumonia: evaluation of low-yield criteria. Ann Emerg Med 1989; 18: 13-20.

30. Heckerling PS, TaPe TG, Wigton RS, Hissong KK, Leikin JB, Ornato JP et al. Clinical prediction rule for pulmonary infiltrates. Ann Intern Med 1990; 113: 664-70.

31. Saldías F, Cabrera D, De Solminihac I, Hernández $P$, GeDERLINI A, Díaz A. Valor predictivo de la historia clínica y examen físico en el diagnóstico de neumonía del adulto adquirida en la comunidad. Rev Méd Chile 2007; 135: 143-52.

32. Pauker SG, Kassirer JP. The threshold approach to clinical decision making. N Engl J Med 1980; 302: 1109-17.

33. Bushyhead JB, Christensen-Szalanski JJ. Feedback and the illusion of validity in a medical clinic. Med Decis Making 1981; 1: 115-23.

34. Niederman MS, Mandell LA, Anzueto A, Bass JB, Broughton WA, CAmpBell GD et al. Guidelines for the management of adults with communityacquired pneumonia. Diagnosis, assessment of severity, antimicrobial therapy, and prevention. Am J Respir Crit Care Med 2001; 163: 1730-54. 
35. British Thoracic Society Standards of Care CommitTEE. British Thoracic Society guidelines for the management of community-acquired pneumonia in adults. Thorax 2001; 56 (suppl IV): 1-64.

36. Sociedad Chilena de ENFERMEDAdes Respiratorias y Sociedad Chilena de InFeCtología. Consenso Nacional 2005: Manejo de la neumonía del adulto adquirida en la comunidad. Rev Chil Enf Respir 2005; 21: 69-140.

37. Emerman CL, Dawson N, Speroff T, Sicilano C, EFFRON D, RASHAD F ET AL. Comparison of physician judgment and decision aids for ordering chest radiographs for pneumonia in outpatients. Ann Emerg Med 1991; 20: 1215-9.

38. Saldías F, Cabrera D, De Solminihac I, Agar V, Díaz A. Evaluación del juicio clínico y las guías de decisión en la pesquisa de pacientes adultos con neumonía adquirida en la comunidad en la unidad de emergencia. Rev Chil Enf Respir 2007; 23: en prensa.

39. Wipf JE, Lipsky BA, Hirschmann JV, Boyko EJ, Takasugi J, Peugeot RL et al. Diagnosing pneumonia by physical examination. Relevant or relic? Arch Intern Med 1999; 159: 1082-7.

40. GonZÁlez MA, Carnicero M, Varela M. Predicción de la presencia de neumonía en el adulto con fiebre. Med Clin (Barc) 1995; 105: 521-4.

41. Lieberman D, Shvartzman P, Korsonsky I, Lieberman D. Diagnosis of ambulatory community-acquired pneumonia. Comparison of clinical assessment versus chest X-ray. Scand J Prim Health Care 2003; 21: 57-60. 